\title{
Q-Factor Prediction and Optimization of Multilayer Inductors for RF Packaging Microsystems Using Time Domain Techniques
}

\author{
Nathan Bushyager, Mekita Davis, Edan Dalton, Joy Laskar, and Manos Tentzeris \\ The Georgia Institute of Technology \\ School of Electrical and Computer Engineering, Atlanta, GA, 30332 USA \\ nbushyager@ece.gatech.edu
}

\begin{abstract}
This paper presents methods using time domain electromagnetic modeling techniques to characterize passive devices in packaging structures. Particularly, the $\mathrm{Q}$ of a novel multilayer inductor is predicted. The inductor uses a "hollow ground plane" configuration to increase performance. The modeling techniques employed include dielectric loss modeling to accurately characterize the device. In addition, several optimizations are used in order to speed computation.
\end{abstract}

\section{Introduction}

The commercial use of RF devices is rapidly increasing. In addition, the complexity of various RF devices is growing in order to augment their functionality and appeal. In contrast to these increased complexity and performance demands, there is a strong market demand for reduced cost. To meet all of these requirements, it is desirable to build microsystems that are complete devices on a single package. Much work has been done in the field of creating passive devices in multilayer technology. One of the major challenges of creating a multilayer inductor in packaging technologies is achieving a reasonably high Q-factor. It is often difficult, expensive, and time consuming to design, build, and test structures with current techniques. This paper demonstrates a method to simulate multilayer inductors, taking into account substrate loss, which can be used to predict the Q-factor, as well as the effective inductance value for the frequencies of operation.

The correct characterization of these components requires that they be examined over the entire frequency band of operation. Time domain techniques are well suited to this requirement as the time domain results from a single simulation can be used to determine the response over an arbitrary frequency band through the use of a Fourier transform. The two techniques that will be discussed in the paper are the finite-difference time-domain (FDTD) [1] technique and the multiresolution time-domain (MRTD) [2-3] technique. A parallel FDTD code can be used to provide fast industry-standard modeling while MRTD can be used to create very efficient highly detailed simulations. By incorporating substrate loss into these two simulations, the Q factor of resonant structures, such as inductors, can be accurately predicted.

The structure that will be simulated in this paper is a multilayer spiral inductor built over a hollow ground plane. The hollow ground plane configuration removes the ground metal directly beneath the spiral and has been shown to increase the Q significantly. The structures will be fabricated using the multilayer organic SOP fabrication facility located at Georgia Tech. The paper will demonstrate how the simulation methods can be used both to model structures and to perform a parametric analysis leading to the optimization of the performance characteristics.

\section{Modeling Methods - Background}

This paper discusses how time domain techniques can be used to characterize and optimize packaging passives such as multilayer spiral inductors. One popular simulator is the finite-difference time-domain (FDTD) technique which has been popular for some time. FDTD has many advantages, the key ones being its relatively simple implementation and high accuracy. These characteristics allow the FDTD user to easily apply several optimizations which can speed execution.

Another technique which is gaining in popularity is the multiresolution time-domain technique (MRTD). MRTD is a powerful electromagnetic simulation technique that takes advantage of multiresolution principles to perform a more efficient simulation than allowed by other techniques. This technique is far newer than FDTD and significantly more complicated, however the benefits that this technique offers are significant.

\section{FDTD}

The finite-difference time-domain (FDTD) method was originally proposed in 1966 [4]. It is a time domain electromagnetic modeling method. There are several advantages of FDTD over other modeling methods. FDTD is derived directly from Maxwell's equations, no approximations are employed. In its basic $2^{\text {nd }}$ order form it uses a Cartesian grid that conforms well to many multilayer structures. Its time domain nature allows it to simulate a wide band in one simulation. These reasons have historically made FDTD a popular choice for many types of simulations.

There are several reasons why FDTD remains popular. There exist many powerful techniques to extend FDTD while keeping the algorithm relatively simple. In addition, it parallelizes extremely well. The FDTD algorithm can provide nearly linear speedup on a variety of supercomputers including workstation clusters such as Linux Beowulf clusters. 
The FDTD code used to simulate the inductors in this investigation makes use of two key optimizations that allow structures to be simulated quickly. The first optimization is a variable grid. This relatively simple optimization over the Yee cell can reduce the number of cells needed for a simulation by an order of magnitude or more. The second optimization is parallelization. The code used in this simulation was optimized to run on a Linux Beowulf cluster.

\section{MRTD}

The multiresolution time-domain (MRTD) technique can be viewed as a generalization of FDTD. MRTD uses multiresolution principles from DSP to discretize electromagnetic fields in both time and space. The basis functions used in this analysis are wavelet functions. These functions have a number of mathematically beneficial characteristics, and have been shown to create very efficient discretizations. Furthermore, MRTD can be used to simulate structures that have varying material properties in a cell. Using wavelet functions, MRTD can simulate many structures more efficiently than FDTD [3].

The modeling of loss in MRTD, however, is difficult. This can be seen by examining Ampere's law:

$$
\frac{\partial \mathbf{D}}{\partial t}=\nabla \times \mathbf{H}-\sigma \mathbf{E}
$$

In FDTD, material properties are constant throughout a cell and $\mathbf{D}$ is transformed into $\varepsilon \mathbf{E}$. The resulting equation can be easily solved for $\mathrm{E}$ and an explicit scheme results [3]. MRTD, however, is not so simple. In lossless materials, an explicit scheme can be found to find each $\mathbf{D}$ coefficient from the $\mathbf{H}$ coeffieients, and then the $\mathbf{E}$ coefficients can be determined by solving a linear system with the $\mathbf{H}$ fields. The coefficients of the $\mathbf{H}$ to $\mathbf{E}$ transformation can be found a priori, however, resulting in a quick scheme. When the D update equations, however, depend on the $\mathbf{E}$ fields there is no simple way to separate the equations, and an implicit scheme results [5].

\section{Multilayer Inductor Modeling}

\section{Device Description}

The inductor modeled in this paper is presented in Figures 1 and 2. In order to give insight into the 3D geometry, a 3D rendering of the structure (with the dielectric removed) is presented. It can be seen that the device is a one port device for characterization, the output port has been connected to ground. The structure is fabricated using two layers of an organic substrate used in the Georgia Tech SOP process. This substrate has an $\varepsilon_{\mathrm{r}}$ of 3.19 and a $\tan \delta$ of 0.026 at $2 \mathrm{GHz}$. The organic substrate is supported by FR4. The ground plane under the turns of the inductor has been removed creating a hollow ground plane configuration. This configuration allows the inductors to have relatively high $\mathrm{Q}$ values.
The total structure is 45 mills thick. The FR4 layer is 40 mills thick, and each layer of the organic substrate is 2.5 mills. The metal, copper, is applied in three layers, one on the FR4 and one on each layer of organic substrate. The metallization thickness varies from layer to layer, and its thickness is significant compared to the layer thickness $(\sim 30 \%)$. The actual device is fed using coplanar waveguide (CPW) probes. The probe grounds were attached to the device ground with vias. In the simulation, the structure is fed with microstrip and the ground plane is expanded to fill the entire computational space (with the exception of the hollow area under the device itself). This allows for both easier modeling and a more accurate representation of how the device will function when combined with other components.

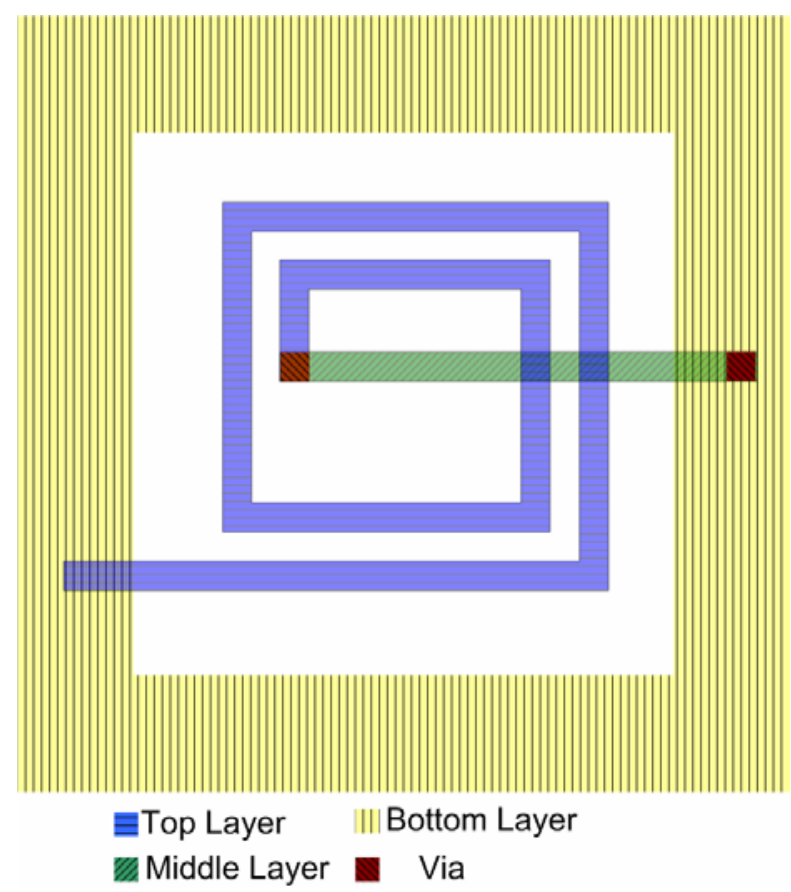

Figure 1: Diagram of modeled structure (metals)

\section{Model}

The device was represented in a Cartesian grid. A variable FDTD grid was used to model the various features of the structure. The depth of the metals was modeled by representing the metals as 'boxes' with a PEC on each side. At least four cells were used across the width of the metal, and two to represent the depth. These requirements determined the remainder of the grid. The feed was elongated to allow the excitation to be performed at a sufficient distance from the inductor without causing interaction.

One important feature to note that is different between the simulated structure and the fabricated structure is the feed. As stated previously the measurement structure is fed with a $\mathrm{CPW}$ probe. The CPW probe grounds were connected to the hollow ground using vias. In the simulated results, the vias are removed. The simulated structure is fed with a microstrip, and thus the ground is extended to the entire space with the exception of the hollow ground area. While the simulated 
structure more closely matched how the device will be used in practice, the lack of grounds most likely effects the results.

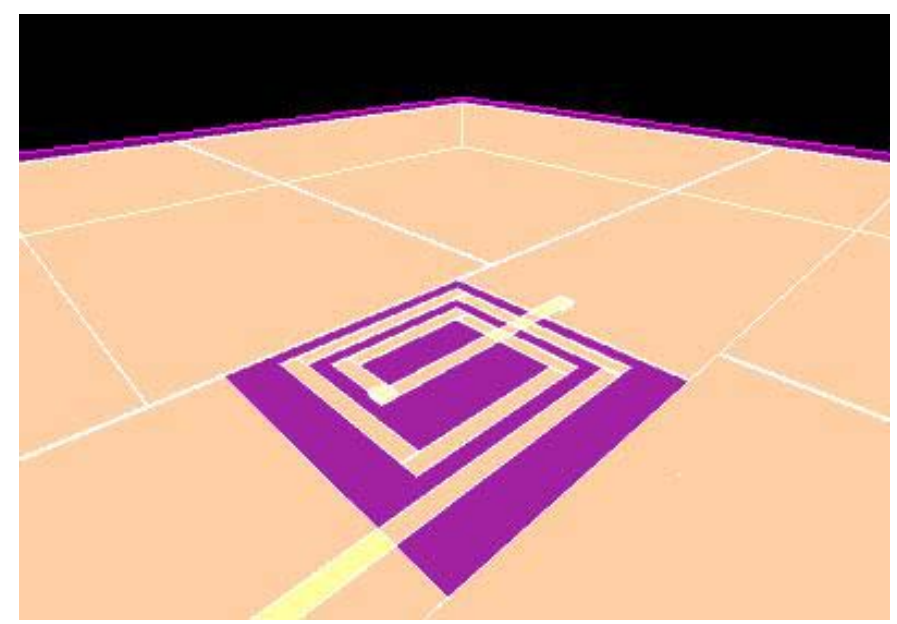

Figure 2: Diagram rendering of modeled structure

The reference plane for both the simulation and the measurement was kept the same. The measurement results are deembedded to the probe tips which are placed at the position the top trace ends in Figure 1. In the simulation, the microstrip fields were probed at this same point in order to determine the voltage.

The simulator used for this experiment was run on eight dual Athlon MP+ 1800 computers, running in parallel. The simulation took approximately 5 hours. A separate simulation was run on a thru line in an identical configuration. Using the thru line as a reference for the input conditions on the inductor, the S-parameters of the device were determined.

From $\mathrm{S}_{11}$ the inductance and impedance of the inductor were determined. $\mathrm{Q}$ was determined by dividing the imaginary part of the impedance by the real part. The same procedures for determining circuit parameters were used in both the simulation and measurement.

\section{Results}

The results for the device of Figure 1 are presented in Figures 3,4, and 5 showing $\mathrm{S}_{11}$, inductance, and $\mathrm{Q}$ for both simulation and measurement. It can be seen that the measurement and simulation results are very similar for all of these components, both in general behavior and in magnitude.

In Figure $3, \mathrm{~S}_{11}$ of the device is shown to be better in simulation than measurement for almost the entire frequency range. This is to be expected as the simulation is of an idealized case with no fabrication and measurement error. At the higher frequencies the results diverge slightly. Another reason for these differences is the slight difference in the simulated and fabricated structures. The simulated structure did not have the vias that existed in the actual structure. This can also be seen to affect the inductance.

Figure 4 shows the effective inductance of the device over the frequency range where $\mathrm{Q}$ is the highest. It can be seen in this case that the measured inductance in this case is actually higher than the simulated inductance. One possible explanation for this is unexplained error, either in simulation, measurement, or fabrication, however, another is the extra vias used to ground the probe pads. These vias may introduce a parasitic inductance that increased the inductance of the device.

In Figure 5, it can be seen that the results for $\mathrm{Q}$ in both simulation and measurement are very similiar. Furthermore, the $\mathrm{Q}$ peaks at approximately the same frequency. The measurement results are not very smooth around the peak showing possible measurement or fabrication error. The $\mathrm{Q}$ value found from simulation is higher than the measurement. This is expected as the simulation of the inductor does not include metal loss. In addition, it was found that the value of $\mathrm{Q}$ is heavily dependent on the loss tangent of the structure. The fabrication process used for these devices is experimental, and thus obtaining a very accurate measurement of loss tangent is difficult. Finally, the via may cause a problem in this case most notably changing the frequency where the $\mathrm{Q}$ becomes negative (the self resonant frequency).

\section{Conclusions}

This paper outlined a method where time-domain modeling techniques can be used to simulate and accurately characterize devices that are traditionally difficult to model. The main technique used was the FDTD technique because of both its simplicity, speed and accuracy. Future investigations may focus on other techniques for comparison.

The results of the simulations show that the $\mathrm{Q}$ of a resonant device, such as an inductor can be predicted using the methods discussed in the paper. Furthermore, acurate prediction can be made of wide-band performance. While it has been shown that there is some disparity between measured and simulated $\mathrm{Q}$ value, the technique can be used to optimize structures. Because measured results match the form of the simulated results, design changes can accurately predict performance changes in the actual device. As such, this technique may be used in the design process to optimize and tune structures to reduce the number of fabrication runs needed to build a component which meets required specifications.

Future work in this area will focus on optimization of these devices and the effects of different feeding methods on their performance. The technique could be used in a design of experiment (DOE) approach to determine the effect of device parameters on performance. 


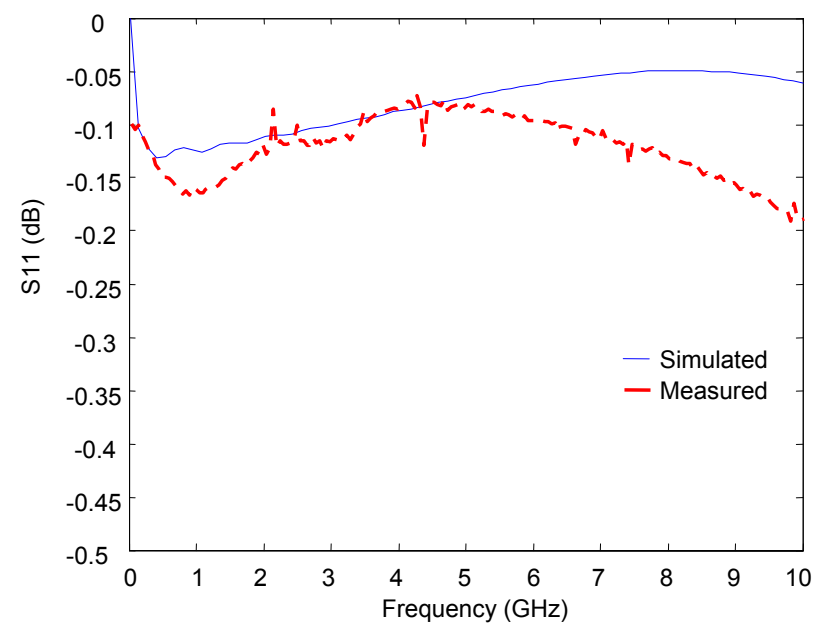

Figure 3: $\mathrm{S}_{11}$ vs. frequency

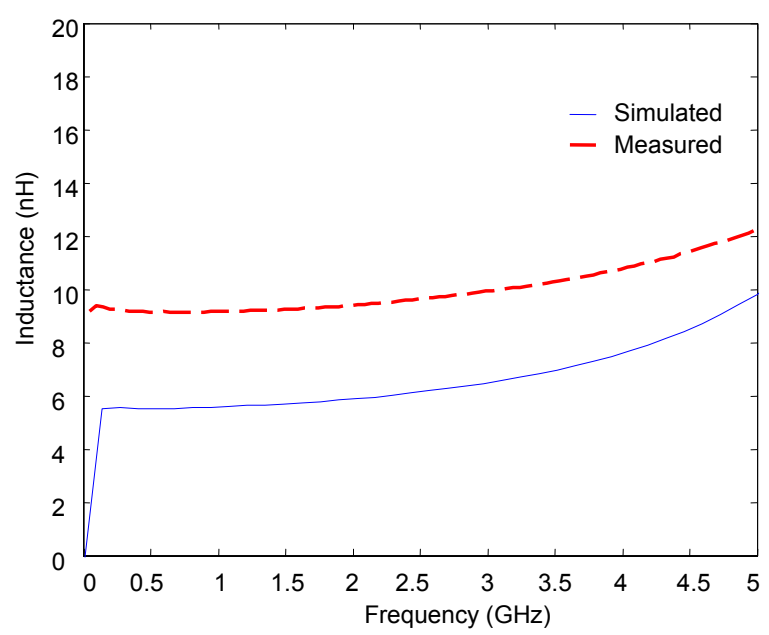

Figure 4: Inductance vs. frequency

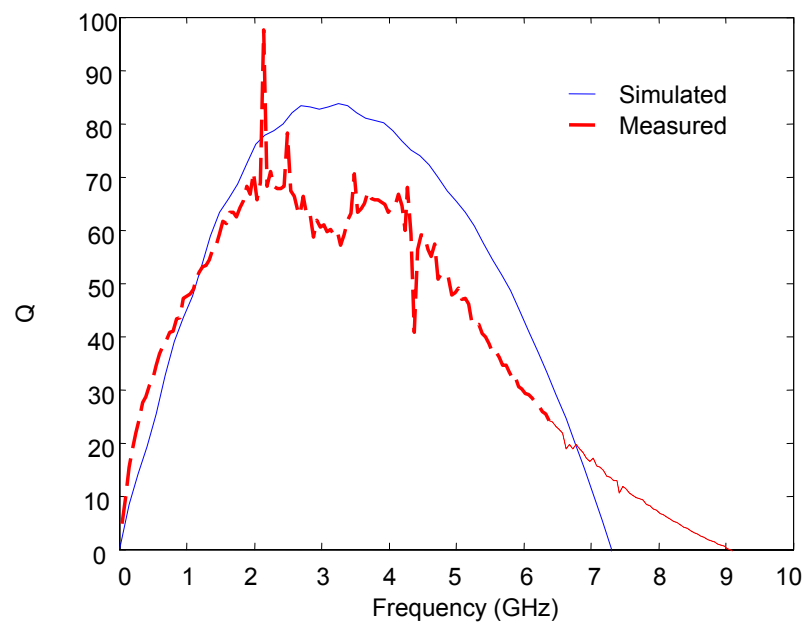

\section{Acknowledgments}

The authors wish to thank the NSF Packaging Research Center at Georgia Tech as well as the NSF CAREER Award and the Yamacraw Initiative of the State of Georgia.

\section{References}

1. A. Taflove and S. Hagness, Computational Electrodynmics: The Finite Difference Time Domain Method, $2^{\text {nd }}$ Ed., Norwood, MA. Artech House, 2000.

2. M. Krumpholz and L.P.B. Katehi, "MRTD: New time domain schemes based on multiresolution analysis," IEEE Trans. Microwave Theory Tech., vol. 44, pp.555-561, Apr. 1996.

3. E. M. Tentzeris, A. Cangellaris, L.P.B. Katehi, J. Harvey, "Multiresoultion time-domain (MRTD) adaptive schemes using arbitrary resolutions of wavelets," IEEE Trans. Microwave Theory Tech., vol. 50, no. 2, pp. 501-516, 2002.

4. K. S. Yee, "Numerical solution of initial boundary value problems involving Maxwe'll's equations in isotropic media," IEEE Trans. Antennas Propagat., vol. AP-14, pp.302-307, 1996.

5. C. D. Sarris, L.P.B. Katehi, and J.F. Harvey, "Mesh truncation methods for the multiresolution time domain technique," Proc. 2000 Antennas and Prop. International Symp., vol. 3, pp. 1350-1353.

Figure 5: Q vs. frequency 